\title{
Exciton spin decay modified by strong electron-hole exchange interaction
}

\author{
G. V. Astakhov ${ }^{1,2}$ * A. V. Koudinov ${ }^{1}$, K. V. Kavokin ${ }^{1}$, I. S. Gagis ${ }^{1}$, Yu. G. Kusrayev ${ }^{1}$, \\ W. Ossau ${ }^{2}$, L. W. Molenkamp ${ }^{2}$, G. Karczewski ${ }^{3}$, T. Wojtowicz ${ }^{3}$, and J. Kossut ${ }^{3}$ \\ ${ }^{1}$ A. F. Ioffe Physico-Technical Institute, Russian Academy of Sciences, 194021 St. Petersburg, Russia \\ ${ }^{2}$ Physikalisches Institut der Universität Würzburg, 97074 Würzburg, Germany \\ ${ }^{3}$ Institute of Physics, Polish Academy of Sciences, 02-668 Warsaw, Poland
}

(Dated: July 7, 2018)

\begin{abstract}
We study exciton spin decay in the regime of strong electron-hole exchange interaction. In this regime the electron spin precession is restricted within a sector formed by the external magnetic field and the effective exchange fields triggered by random spin flips of the hole. Using Hanle effect measurements, we demonstrate that this mechanism dominates our experiments in $\mathrm{CdTe} /(\mathrm{Cd}, \mathrm{Mg}) \mathrm{Te}$ quantum wells. The calculations provide a consistent description of the experimental results, which is supported by independent measurements of the parameters entering the model.

PACS numbers: 72.25.Rb, 72.25.Fe, 78.67.-n
\end{abstract}

Spin is a fundamental invariant of an electron. In semiconductors, the electron and hole spins can be optically oriented by a circularly polarized light [1]. Recently much interest has been attracted to this field caused by possible applications (spintronics) 2]. In particular, the spin trapped in a quantum dot has been proposed to serve as a qubit in the quantum computation schemes [3] using an all-optical protocol for spin readout and initialization [4]. Therefore, a detailed understanding of the spin decay processes are of crucial importance for the functionality of spin-based devices.

The most widespread method for studying the dynamics of the non-equilibrium spin populations in semiconductors is optical orientation [1]. Circularly polarized light excites electrons with a predominant spin polarization along the direction of the incident light beam. When an external magnetic field $B$ is applied in Voigt geometry, i.e., in the plane of the sample, the optically oriented electron spins start precessing with Larmor frequency $\Omega=g_{e} \mu_{B} B$ around the field direction $\left(g_{e}\right.$ is the electron g-factor). Under continuous wave (cw) excitation this results in a decrease of the spin mean value with growing $B$, such a behavior is known as the Hanle effect 1]. The half width at half maximum (HWHM) of the Hanle curve at $B=B_{1 / 2}$ corresponds to the condition $\Omega T_{s e}=1$, and hence allows determination of the electron spin lifetime $T_{s e}$. For optically created electrons it is governed by the electron spin relaxation time $\tau_{s e}$ and the lifetime time $\tau$ of the electron-hole pair (exciton): $T_{s e}^{-1}=\tau_{s e}^{-1}+\tau^{-1}$.

This classical picture will be modified drastically by isotropic electron-hole (eh) exchange interaction. It manifests itself as a splitting $\Delta_{0}$ between the radiative $( \pm 1)$ and nonradiative $( \pm 2)$ doublets of the neutral exciton [5]. The electron spin now precesses in a total field formed by the external magnetic field $B$ and the exchange field of the hole $B_{e x}=\Delta_{0} /\left|g_{e}\right| \mu_{B}$. Moreover, due to the hole spin relaxation, the exchange field $B_{e x}$ changes with time introducing additional difficulties for the theoretical de- scription. The influence of the eh-exchange interaction [6, 7] and the importance of the hole spin relaxation rate on the electron spin decay $[\underline{8},[9]$ have been recognized in previous theoretical works. However, the Hanle effect in the regime of the strong electron-hole exchange coupling, when the frequencies associated with the exchange interaction become greater than relaxation rates of electrons and holes, has not been investigated so far. The role of the exchange coupling is especially high in systems with reduced dimensionality like quantum dots (QDs) or quantum wells (QWs), where the exciton is confined in a small volume.

In this Letter we consider exciton spin decay in the regime of strong eh-exchange interaction. We demonstrate that in this limit the classical picture is not valid any more. While the Hanle curve still originates from depolarization of the electron spin in an external magnetic field, it occurs in a rather different manner and $B_{1 / 2}$ now depends on the eh-exchange splitting $\Delta_{0}$, the hole spin relaxation time $\tau_{s h}$, and the exciton life time $\tau$. We provide strong experimental evidence by comparing the Hanle curves of a single $\mathrm{CdTe} /(\mathrm{Cd}, \mathrm{Mg}) \mathrm{Te} \mathrm{QW}$ detected at the neutral exciton $\left(\mathrm{X}^{0}\right)$ and positively charged trion $\left(\mathrm{T}^{+}\right)$, where in the latter case the eh-exchange interaction is suppressed. We find that the characteristic depolarization field of the exciton Hanle curve $B_{1 / 2}^{X}$ is an order of magnitude larger than that of the trion Hanle curve $B_{1 / 2}^{T}$, indicating their different physics.

We now consider schematically the regime of strong ehexchange interaction following the ideas of Ref. 10 and discuss the limitations of this approach (see Fig. 1). Let as an example circularly polarized light excite exciton with the z-projection of the angular moment $J_{z}=-1$, consisting of an electron with spin up $(+1 / 2)$ and a hole with spin down $(-3 / 2)$. How will this system evolve in a magnetic field $B$ directed perpendicular to the quantization axis (Fig. 11)?

First, we assume that the in-plane hole g-factor is close to zero. This is valid when the heavy-hole band is split off 


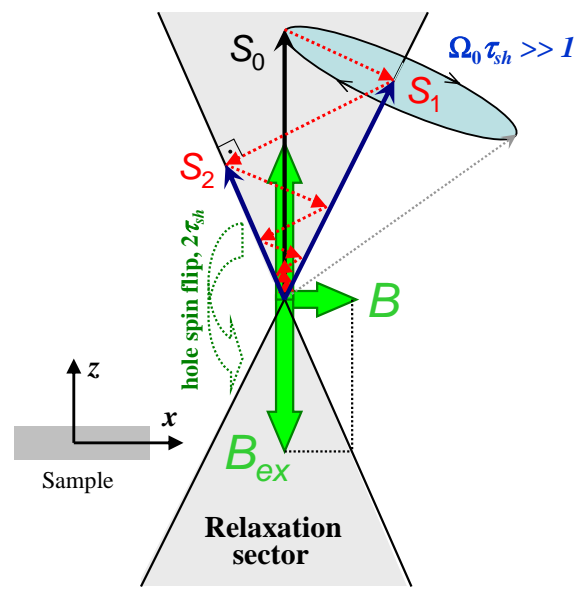

FIG. 1: (Color online) Chain of the electron spin decay $\left(S_{0} \rightarrow\right.$ $\left.S_{1} \rightarrow S_{2} \ldots\right)$ within the relaxation sector shown by gray area. Such a chain represents the evolution of the mean spin of the electron for strong exchange interaction $\left(\Omega_{0} \tau_{s h} \gg 1\right)$. The relaxation sector is formed by the two fields acting on the electron: The exchange field of the hole $B_{e x}$ and the external magnetic field $B$.

from the light hole band as indeed occurs in low dimensional systems, and when their mixing may be neglected. We also ignore the anisotropic exchange interaction [5]. Under this assumption the hole spin is always directed parallel to the $\mathrm{z}$-axis (perpendicular the sample plane), and the spin dynamics the hole is capable of are random spin flips with a mean time between the successive flips of $2 \tau_{s h}$. In other words, this applies a hole spin relaxation with a characteristic time $\tau_{s h}$.

The electron, possessing a nonzero g-factor, experiences the action of the external field $B$ and of the exchange field of the hole $B_{e x}=\Delta_{0} /\left|g_{e}\right| \mu_{B}$, which in our example is initially directed upwards (Fig. 1). The electron spin precesses in the resulting total field, the condition of strong eh-exchange implying that the Larmor frequency $\Omega_{0}=\Delta_{0} / \hbar$ is so high that the electron spin makes at least one turn around the total field before the precession is interrupted by a flip of the hole spin. Formally, this condition reads $\Omega_{0} \tau_{s h}>1$. If this is fulfilled, only the projection $S_{1}$ of the initial mean electron spin $S_{0}$ on the direction of the total field is conserved until the moment of the hole spin flip, while the transverse components of the electron spin become random (Fig. 1).

The above process is only a first link in the chain of the spin evolution of the exciton. The flip of the hole spin results in the inversion of the direction of the exchange field; the direction of the total field will consequently change and the electron spin precession will proceed around the new direction (see Fig. 1). Analogously to the previous consideration, the precession will lead to a transformation of the mean spin $S_{1}$ into $S_{2}$. After that, the new flip of the hole spin will return the total field to the initial direction. The next step will result in the de- cay of the new portion of the electron spin, etc. (Note that we implicitly assume a long electron spin relaxation time $\tau_{s e}>\tau_{s h}$.) The cascade process of the spin depolarization of the electron will last until it is interrupted by excitonic recombination with a characteristic time $\tau$.

As a result of the above deliberation, we can introduce a relaxation sector shown by the gray area in Fig. 1] which lies in the plane formed by $B$ and $B_{e x}$ fields. The relaxation sector restricts the directions of the electron spin and determines its decay path. This picture is thus valid for zero in-plane hole g-factor, and provided $1 / \Omega_{0}<\tau_{s h}<\tau_{s e}, \tau$. As we show in the following, these conditions can indeed be realized experimentally.

The sample under study was grown by molecular beam epitaxy on a (001) GaAs substrate. The structure consists of a single (38- $\AA$-wide) $\mathrm{CdTe} / \mathrm{Cd}_{0.7} \mathrm{Mg}_{0.3} \mathrm{Te} \mathrm{QW}$ and is nominally undoped. The photoluminescence (PL) was excited by a dye laser with tunable photon energy and detected with a 1-m spectrometer and a photomultiplier tube. In order to detect the polarization of the emission we used a standard scheme with a piezo-elastic modulator and a two-channel photon counter. Magnetic field was applied either perpendicular to the sample plane (Faraday geometry) or in the sample plane (Voigt geometry). All experiments were carried out at a temperature $1.6 \mathrm{~K}$.

A typical PL spectrum is shown in Fig. 2(b). Generally, the spectrum consists of two bands. The high-energy peak is attributed to the neutral exciton $\mathrm{X}^{0}$, and the lowenergy one is ascribed to a charged exciton, or trion, $\mathrm{T}$ 11]. Our analysis shows that we are dealing with the positively charged trion $\mathrm{T}^{+}$12]. It consists of one electron and two holes. The holes form a spin singlet with zero total spin $s_{h}=0$ as shown in Fig. 33(a), so the optical orientation signal from $\mathrm{T}^{+}$always reflects the spin polarization of the unpaired electron $s_{e}$. Moreover, for the same reason (i.e., $s_{h}=0$ ) the exchange field is zero for a $\mathrm{T}^{+}$trion, $B_{e x}=0$, which allows us to study the electron spin relaxation free of exchange interaction.

Figure 2(a) shows a typical spectrum of the optical orientation under quasi-resonant excitation at the trion line. While quite high in absence of external field, the optical orientation signal approaches zero already at a relatively low magnetic field of $B=0.5 \mathrm{~T}$. The Hanle curve detected at the PL maximum is given in Fig. 22 (c) by squares. It has a Lorentzian shape and is perfectly described by the classical formula derived for the free spin precession of an electron [1]

$$
P_{T^{+}}=2 s_{e}=\frac{T_{s e}}{\tau_{r}} \frac{2 s_{e 0}}{1+\left(B / B_{1 / 2}^{T}\right)^{2}},
$$

with $s_{e}$ being its mean spin value and

$$
B_{1 / 2}^{T}=\frac{\hbar}{\left|g_{e}\right| \mu_{B} T_{s e}} \text { and } \frac{1}{T_{s e}}=\frac{1}{\tau_{r}}+\frac{1}{\tau_{s e}} .
$$

Here $s_{e 0}$ is the initial value of the electron spin as created at the $\mathrm{T}^{+}$state by optical orientation. The best fit to the 

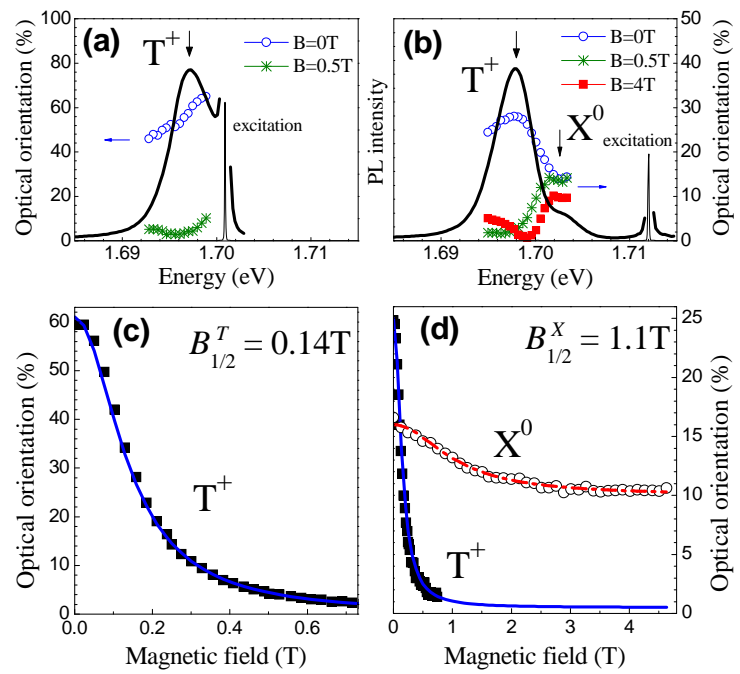

FIG. 2: (Color online) Spectra of PL and optical orientation for quasi-resonant excitation at the positively charged trion (a) and non-resonant excitation above the neutral exciton (b). (c) The Hanle effect measured for the trion $\left(\mathrm{T}^{+}\right)$at the same excitation conditions as in panel (a). Solid line is the fit using Eq. (1) with characteristic field $B_{1 / 2}^{T}=0.14$ T. (d) The Hanle effect measured for the exciton $\left(\mathrm{X}^{0}\right)$ at the same excitation conditions as in panel (b). Dashed line is the fit using Eqs. (6) and (7) with characteristic field $B_{1 / 2}^{X}=1.1 \mathrm{~T}$. Magnetic fields are applied in Voigt geometry.

data in Fig. 2(c) is achieved with $2 s_{e 0} T_{s e} / \tau_{r}=0.61$ and $B_{1 / 2}^{T}=0.14 \mathrm{~T}$ (solid line). Taking the electron g-factor $g_{e}=-1.3$ known from the spin-flip Raman scattering experiments [13] and the obtained value of $B_{1 / 2}^{T}$, we deduce for the electron spin lifetime $T_{s e}=61 \mathrm{ps}$. Assuming that the conditions of quasi-resonant excitation are close to the ideal case $\left(s_{e 0} \approx 1 / 2\right)$, one can estimate a recombination time of $\tau_{r}=100 \mathrm{ps}$. This value is in agreement with typical decay times of the trion PL obtained from direct time-resolved experiments on CdTe-based QWs 14]. In the case of nonresonant excitation [Fig. 2(b)] the HWHM of the trion Hanle curve [Fig. 2(d)] remains unchanged, again yields $B_{1 / 2}^{T}=0.14 \mathrm{~T}$ [Fig. 2(d)]. Reduction of the initial electron spin $\left(s_{e 0}<1 / 2\right)$ is most likely caused by relaxation processes to the trion ground state.

In contrast to the positively charged trion, the neutral exciton $\mathrm{X}^{0}$ displays a rather different behavior [see Fig. [2(d)]. First, while the magnetic field is increased, the polarization does not disappear but saturates at a nonzero level of $\sim 0.1$. Second, while there exists a field-dependent part of polarization which is well fitted by a Lorentzian curve, analogously to the case of the trion, the characteristic field of this depolarization equals $B_{1 / 2}^{X}=1.1 \mathrm{~T}$, much larger than that of the $\mathrm{T}^{+}$.

Give the above discussion, the natural explanation of this observation is that the wide depolarization curve of the exciton originates from the exchange interaction between the electron and the hole (as in Fig. 11). In or-
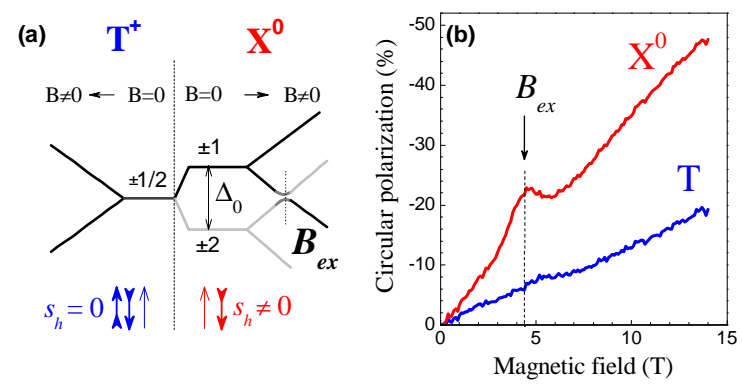

FIG. 3: (Color online) (a) Energy diagrams of the trion $\left(\mathrm{T}^{+}\right)$ and exciton $\left(\mathrm{X}^{0}\right)$ spin levels in a magnetic field applied in Faraday geometry. In the studied samples the isotropic exchange splitting is $\Delta_{0}=\hbar \Omega_{0} \approx 0.33 \mathrm{meV}$. For an external magnetic field $B=B_{e x}$ the radiative (black) and nonradiative (gray) branches exhibit anti-crossing. (b) Circular polarization of the exciton $\left(\mathrm{X}^{0}\right)$ and the trion $(\mathrm{T})$ emission induced by the magnetic field at a non-resonant excitation (above the QW barrier). At $B_{e x} \approx 4.5 \mathrm{~T}$ a distinct anomaly is observed.

der to examine this we measured the exchange splitting $\Delta_{0}$ independently. Following scheme shown in Fig. 3(a), a magnetic field $B$ applied in the Faraday configuration may lead at some field values to an anti-crossing of the bright and dark exciton states [15]. The first anti-crossing occurs when $\left|g_{e}\right| \mu_{B} B=\Delta_{0}$, and hence this magnetic field $B=B_{e x}$ may be interpreted without any additional assumption as the exchange field of the hole acting on the electron . Experimentally, the anti-crossing manifests itself as an anomaly in the polarization of the PL 16, 17]. Indeed, we observed such an anomaly (at $B_{e x} \approx 4.5 \mathrm{~T}$ ) when detect circular polarization degree of the exciton in emission [Fig. 3(b)]. Thus we have the following relationship between characteristic magnetic fields $B_{1 / 2}^{T} \ll B_{1 / 2}^{X} \ll B_{e x}$.

We now demonstrate that the experimental behavior is in good agreement with an analytical solution for the model presented in Fig. 1] Assuming $\tau_{s h} \ll \tau$ the electron depolarization essentially occurs for weak external fields $B \ll B_{e x}$. So the relaxation sector shown in gray in Fig. 1 is narrow, and the decrease of the electron spin density $S_{e}$ per single flip of the hole spin approximately equals $2 S_{e}\left(B / B_{e x}\right)^{2}$. The master equation for $S_{e}$ then takes a simple form

$$
\dot{S}_{e}=s_{e 0} G-\frac{S_{e}}{\tau}-\frac{S_{e}}{\tau_{s h}}\left(\frac{B}{B_{e x}}\right)^{2} ;
$$

here the first term describes the spin creation with the excitation rate $G$ (with $s_{e 0} \leq 1 / 2$ phenomenologically accounting for possible losses of the electron spin polarization in the higher-lying state where the exciton is actually created), the second term represents the losses by excitonic recombination, the third term describes the decrease of spin via the chain process of Fig. 1 $\left[\left(2 \tau_{s h}\right)^{-1}\right.$ has the sense of number of hole spin flips per unit of time]. Setting $\dot{S}_{e}$ to zero, one can find the steady-state value of 
$S_{e}$ as a function of $B$

$$
S_{e}=s_{e 0} \frac{G \tau}{1+\frac{\tau}{\tau_{s h}}\left(\frac{B}{B_{e x}}\right)^{2}} .
$$

When $\tau_{s h} \ll \tau$, the correlation between the electron and hole spins is weak, so the intensities of the components of the luminescence with opposite circular polarizations ( $I_{+}$ and $I_{-}$) depend only on the mean spin values $s_{e}=S_{e} / G \tau$ and $s_{h}=-\frac{3}{2} \tau_{s h} / \tau$ :

$$
I_{ \pm} \propto \frac{1}{3}\left(\frac{1}{2} \pm s_{e}\right)\left(\frac{3}{2} \mp s_{h}\right) .
$$

Using once more that $\tau_{s h} / \tau$ is small, we finally obtain the luminescence polarization:

$$
P_{X^{0}} \approx \frac{\tau_{s h}}{\tau}+\frac{2 s_{e 0}}{1+\frac{\tau}{\tau_{s h}}\left(\frac{B}{B_{e x}}\right)^{2}} .
$$

The first (field-independent) contribution originates from the mean spin polarization of holes. Its value is controlled by the ratio of the hole spin relaxation time and the exciton lifetime. The inverse of this ratio also enters the second term describing the chain spin depolarization of electrons.

We now compare the result of the calculation with the experimental data. The ratio of the hole spin relaxation time and the exciton lifetime can be estimated from the high-field value in Fig. 2(c), $\tau_{s h} / \tau \approx 0.1$. This estimate looks reasonable, as for CdTe-based QWs the typical reported values of the hole spin relaxation time are on the order of tens of picoseconds 18]. Together with the value of the exchange field $B_{e x} \approx 4.5 \mathrm{~T}$ taken from the experimental data in Fig. 3(b), this gives a Lorentzian depolarization curve with a HWHM of $1.4 \mathrm{~T}$, in reasonable agreement with the experimentally measured value $B_{1 / 2}^{X}=1.1 \mathrm{~T}$. It turns out that in the strong exchange interaction regime, multiple flips of the hole spin result in a narrowing of the depolarization curve by a factor $\sqrt{\tau / \tau_{s h}} \approx 3.2$ as compared to the field of the exchange interaction:

$$
B_{1 / 2}^{X}=B_{e x} \sqrt{\tau_{s h} / \tau} .
$$

Equation (17) summarizes our key result. Note that if the exciton lifetime $\tau$ is controlled by the radiative recombination time $\tau_{r}$, as for the trion $\left(\tau \approx \tau_{r}=100 \mathrm{ps}\right)$, then $\tau_{s h} \approx 10 \mathrm{ps}$ and the condition of strong exchange interaction is reasonably satisfied, $\Omega_{0} \tau_{s h}=5$.

In summary, by studying the depolarization of the PL from of the positive trion and from the exciton in a CdTe QW, we were able to investigate the exciton spin decay subject to strong exchange interaction. The spin of an electron interacting with a hole precesses in tilted magnetic fields, trigged by the random spin flips of the hole. This process results in a depolarization pattern with the characteristic field $B_{1 / 2}^{X}=\Delta_{0} \tau^{1 / 2} /\left|g_{e}\right| \mu_{B} \tau_{s h}^{1 / 2}$, which was experimentally observed and verified by separate determination of $\Delta_{0} /\left|g_{e}\right| \mu_{B}$ and $\tau^{1 / 2} / \tau_{s h}^{1 / 2}$. The reported mechanism is of interest for nanostructures with sizable isotropic electron-hole exchange interaction (e.g., $\Delta_{0} \approx 0.33 \mathrm{meV}$ in this work).

The study was supported by INTAS (03-51-5266) as well as by Russian Foundation for Basic Research and SFB 410.

* E-mail: astakhov@physik.uni-wuerzburg.de

[1] Optical Orientation, edited by F. Meyer and B. P. Zakharchenya (North-Holland, Amsterdam, 1984).

[2] G. A. Prinz, Science 282, 1660 (1998).

[3] D. Loss and D. P. DiVincenzo, Phys. Rev. A 57, 120 (1998).

[4] A. Shabaev, Al. L. Efros, D. Gammon, and I. A. Merkulov, Phys. Rev. B 68, 201305(R) (2003).

[5] E. L. Ivchenko and G. E. Pikus, Superlattices and Other Heterostructures: Symmetry and Optical Phenomena, Springer-Verlag, Berlin, 1997.

[6] M. I. Dyakonov and V. I. Perel, Zh. Eksp.Teor. Fiz. 65, 362 (1973).

[7] G. L. Bir and G. E. Pikus, Zh. Eksp. Teor. Fiz. 64, 2210 (1973).

[8] M. I. Dyakonov and V. I. Perel, Zh. Eksp.Teor. Fiz. 60, 1954 (1971).

[9] M. Dyakonov, X. Marie, T. Amand, P. Le Jeune, D. Robart, M. Brousseau, J. Barrau, Phys. Rev. B 56, 10412 (1997).

[10] I. S. Gagis, K. V. Kavokin, and A. V. Koudinov. Proc. 13th Int. Symposium "Nanostructures: Physics and Technology", St.- Petersburg, 2005. Ioffe Institute, 2005, p.350.

[11] K. Kheng, R. T. Cox, Y. M. d'Aubigne, F. Bassani, K. Saminadayar, and S. Tatarenko Phys. Rev. Lett. 71, 1752 (1993); G. Finkelstein, H. Shtrikman, and I. BarJoseph, Phys. Rev. Lett. 74, 976 (1995); G. V. Astakhov, D. R. Yakovlev, V. P. Kochereshko, W. Ossau, J. Nürnberger, W. Faschinger, and G. Landwehr, Phys. Rev. B 60, 8485(R) (1999).

[12] Identification whether the trion is positively or negatively charged is based on experimental value of the characteristic magnetic field $B_{1 / 2}^{T}=0.14 \mathrm{~T}$ [Fig[2)]. The Hanle effect detected on the trion line at a quasi-resonant excitation must arise from the precession of spin of the unpaired particle. That particle can not be a hole, as the hole possesses too small in-plane g-factor $g_{h \perp}<0.04$ [13] resulting in the spin lifetime $T_{s}>2 \mathrm{~ns}$. The latter value is clearly meaningless in view of typical recombination times $\sim 100$ ps reported for similar QWs in many papers. So the particle responsible for the low-energy PL line must be positively charged trion $\mathrm{T}^{+}$which includes unpaired electron.

[13] A. A. Sirenko, T. Ruf, M. Cardona, D. R. Yakovlev, W. Ossau, A. Waag, and G. Landwehr, Phys. Rev. B 56, 2114 (1997).

[14] V. Ciulin, P. Kossacki, S. Haacke, J.-D. Ganiere, B. De- 
veaud, A. Esser, M. Kutrowski, and T. Wojtowicz, Phys. Rev. B 62, 16310(R) (2000).

[15] E. L. Ivchenko, A. Yu. Kaminskii, Phys. Solid State 37, 768 (1995); Fiz. Tverdogo Tela 37, 1418 (1995).

[16] N. G. Romanov, I. V. Mashkov, P. G. Baranov, P. Lavallard, R. Planel, JETP Lett. 57, 802 (1993); Pis'ma JETP 57, 788 (1993).
[17] Yu. G. Kusrayev, B. P. Zakharchenya, G. Karczewski, T. Wojtowicz, and J. Kossut, Solid State Commun. 104, 465 (1997).

[18] E. Vanelle, M. Paillard, X. Marie, T. Amand, P. Gilliot, D. Brinkmann, R. Levy, J. Cibert, and S. Tatarenko, Phys. Rev. B 62, 2696 (2000). 